\title{
Autonomous WSN for Lawns Monitoring in Smart Cities
}

\author{
José Marín ${ }^{1}$, Javier Rocher ${ }^{2}$, Lorena Parra ${ }^{2}$, Sandra Sendra ${ }^{2,3}$, Jaime Lloret ${ }^{2}$, Pedro V.Mauri ${ }^{1}$ \\ ${ }^{1}$ IMIDRA. Finca “El Encin”. A-2, Km 38,2. 28800 Alcalá de Henares, Madrid, Spain \\ ${ }^{2}$ Instituto de Investigación para la Gestión Integrada de zonas Costeras. Universitat Politècnica de València, \\ C/Paraninf, 1. 46730. Grao de Gandia, Valencia, Spain \\ ${ }^{3}$ Departamento de teoría de la señal, telemática y comunicaciones, ETS Ingenierías Informática y de Telecomunicación. \\ Universidad de Granada \\ C/ Periodista Daniel Saucedo Aranda, s/n. E-18071 Granada, Spain \\ jmarin@areaverde.es, jarocmo@alumni.upv.es, loparbo@doctor.upv.es, ssendra@ugr.es, jlloret@dcom.upv.es, \\ pedro.mauri@madrid.org
}

\begin{abstract}
The urban lawns are frequently composed by different grass species to combat some problems of water scarcity and diseases. In order to maintain these lawns, high amount of water is required. Nowadays, smart cities can be understood as a new concept of city that includes, among others, efficient distribution of energy, water, and other resources by using technology. In these cases, the main challenge is to try to estimate the necessary amount of water for irrigation and the phytosanitary uses without wasting water. In this paper, we propose a method to identify the percentage of grass coverage in lawns to deduce the grass productivity and estimate the most accurate quantity of water to ensure a good production of grass. The system is based on a Smart Autonomous Vehicle (SAV) controlled by an Arduino Mega 2560. It also contains an array of 120 colour sensors used to gather the data. The selected colour sensor is a TCS3472. With these sensors, we obtain the RGB histograms of the lawns. For these experiments, we have several lawn parcels of $1.5 \times 1 \mathrm{~m}$. From these, a matrix of $150 \times 100 \mathrm{RGB}$ values is obtained. After processing the green values of matrix, we have observed a correlation between the level of coverage and these values. The grass coverage is related with values of brightness between 40 and 60 which allow us to classify the lawn as a function of its coverage and the irrigation needs.
\end{abstract}

Keywords-wireless sensors network (WSN); smart city; urban lawns; water sustainability; RGB sensor

\section{INTRODUCTION}

In the urban lawns different grass species are used and combined. High amount of water is required to maintain those lawns. The freshwater is a limited resource where only the $2.5 \%$ of the total worldwide water is freshwater [1]. Less than $1 \%$ of this water can be used for irrigation, industry process, human consumption, etc [2]. In addition, the increase of population and the global warming are worsen this problem [3].

The smart cities meant to be a new model of city characterized by an efficient distribution of energy, water, and other resources. For this purpose the use of technology is the key [4]. These smart cities pretend to achieve a sustainable natural resources usability by employing the information and communication technologies (ICTs). Several authors point the need of ICTs to manage the water acquisition model and the water distributions $[5,6]$. The smart city concept may be a solution to optimize the urban lawns irrigation.

Recently, several authors point out solutions for precision gardening [7-9]. While some authors stand up for using cameras and plant recognition systems [7, 9], others prefer the use of soil moisture sensors [8] to define the irrigation needs. IN any case, it is necessary to find methods in order to automatize the gardening supervision and optimize the irrigation tasks.

The solution to this problem seems to be the use of Wireless Sensor Networks (WSN). WSNs are being widely used for monitoring and optimizing process in farming [10, $11]$, agriculture [12, 13] and water distribution [14]. For this reason, we propose the use of a WSN for lawns monitoring.

Thus, this paper proposes to include the urban lawns monitoring into the smart city concept. Our aim is to adjust the amount of water assigned for irrigation and the phytosanitary products. We propose a method to identify the coverage of grass and to deduce the grass productivity. The proposed system is based on RGB sensors. The information gathered by the RGB sensors is used to distinguish between grass or soil coverage. The node used to process these data is an Arduino Mega 2560. In addition, we use an array of 100 RGB sensors to gather the data. The used sensor is the TCS3472. This node is included in a smart autonomous vehicle (SAV) which is included in a WSN. The SAV moves along the lawn to gather the data. These data is statistically analysed to determine the water requirements of each kind of lawn. The results shows that for this combination of grass, we should take the green values of a picture to determine the level of coverage of an area.

The rest of paper is structured as follow. Section II presents the state of art. The hypothesis in which our work is based is presented in Section III. Section IV explains the architecture of our autonomous WSN for lawns monitoring. The results are 
exposed and discussed in Section V. Finally, the Section VI summarizes the conclusion and future work.

\section{RELATED WORK}

In recent years, the use of electromagnetic radiation in the visible range of spectrum and infra-red can be useful for monitoring the environment status. This section presents some works about how to estimate the water needs of lawns and gardens as a function of the data gathered by sensors and pictures.

Gardening is currently being modernized with several methods. An interesting proposal presented by Kumar et al. [15] is based on a Smart Autonomous Gardening Rover that is able to identify and classify different species of plants using extraction algorithms and a neural network. Once the plant is identified the rover introduces its arm containing the sensors and according to the measurements it takes it sprays water and fertilizers from this arm.

Another simpler system is developed by Tripathy et al. [16] This system is used for monitoring the demands of water in the garden using low cost sensors. The sensor senses the light, temperature and water in the soil. With these parameters the system determines the quantity of water the plants need.

Photographic analysis can be used for determining the frost damage in a crop. Macedo-Cruz et al. [17] take photographs with a camera with CCD technology and use a combination of three thresholding strategies (the Otsu method, the isolate algorithm and the fuzzy thresholding method) for determining the frost damage.

One example of the assembly of a sensor based on cameras is the one presented by Yang [18]. In this paper, authors study the placement of 4 cameras in an airplane that capture images in blue $(430-470 \mathrm{~nm})$, green $(530-570 \mathrm{~nm})$, red $(630-670 \mathrm{~nm})$ and near-infrared (NIR, $810-850 \mathrm{~nm}$ ). The researchers results shows that the system could be used to monitor crop pest and growing conditions, mapping invasive weeds and assessing wetland ecosystems.

WSNs can be used for image analysis. Lloret et al. [19] designed a WSN for detecting any unusual status in the leaves of a vineyard. In this proposal, the camera takes images and the sensor node process these images to determine if some anomalies are present in the leaves. When an anomaly is detected the sensor nodes send a message to a sink node and this warns the person in charge of this wine exploitation.

Another example of WSN is presented by Garcia-Sanchez et al. [20]. They propose a sensors network for measuring important parameters for agriculture. The system is based on cameras and infrared sensors to detect the presence of intruders in fields. Each group of sensors send the information to a Gateway which sends this information to the farmer cooperative and from there the farmers are renamed. As wireless technology, authors uses IEEE 802.15.4 standard which is able to reach distances up to $7 \mathrm{~km}$.

The use of drones is currently a very popular method to obtain aerial images. Candiago et al. [21] uses a drone equipped with a Tetracam ADC Micro camera for acquiring images in the red (R), green (G) and near-infrared (NIR) bands, allowing to calculate some of the most common vegetation indices, i.e., the normalized difference vegetation index (NDVI), the Green Normalized Difference Vegetation Index (GNDVI) and the Soil Adjusted Vegetation Index (SAVI). With their work, they demonstrate the great potential of drones in precision agriculture

Another example of the use of drones with cameras is the proposal presented by Cambra et al. [22]. Authors presented a network (with an ad hoc communication protocol) consisting of a drone, several sensors and pressure sprayer. The network, by means of the videos captured by drone and data from sensors, enables a set of sprayers in a determined area with weeds. This video is transferred to a $\mathrm{PC}$ which will perform an analysis of the same through the OpenCV library.

García et al. [23] use the analysis of satellite photography captured by Quickbird. The different wavelength images are used to obtain the vegetation indexes green, NIR, NDVI, panchromatic and RVI indices to characterize the size and potential of each olive.

The use of a soil moisture sensor has been ruled out because these indicate the water in the soil but not the moisture of the grass which was our main objective. The use of drones cannot be used in this case because the system is used to monitor the grass in parks and public gardens. Flying a drone in these places can be dangerous for pedestrians and the Spanish law does not allow it [24]. The use of satellite images cannot be used because of the gaps in this technology (low precision and high round trip time to the same point [25]). Thus, our proposal presents a vehicle able to take values of RGB levels of grass and decide the amount of water the lawn needs

\section{TEST BENCH}

This section explains the hypothesiswe base our experiments to find the relation between RGB values and the level of grass coverage. The objective of this analysis is to detect the loss in grass coverage and the need of water.

\section{A. Hypothesis}

A previous test [13] has demonstrated that the blue values of a RGB sensor are correlated with the quantity of grass (in $\mathrm{g} / \mathrm{m}^{2}$ ). In that study, we analyzed regions of grass with high coverage $(\approx 100 \%)$. In this case, we will compare three kind of regions, i.e., regions with high coverage $(\mathrm{HC})(\approx 100 \%)$, regions with low coverage $(\mathrm{LC})(\approx 50 \%)$ and regions with very low coverage (VLC) $(<<50 \%)$. Our thesis supposes that the different coverages (grass or soil) will produce different output values in the RGB sensor. The expected differences are the following:

- The data from regions with high coverage will present lower values in Red than the regions with low coverage.

- The data from regions with high coverage will present higher values in green than the regions with low coverage. 
- $\quad$ The data from regions with high coverage will present lower values in blue than the regions with low coverage (as we can see in [13]).

To perform our study, we distinguish between three groups of coverage, areas with HC (See Fig. 1a), areas with LC (See Fig. 1b) and areas with VLC (See Fig. 1c). In our previous study we obtained a mathematical model for each species. The current study is not focuses on determined grass species. In fact, we use a combination of several species and we only analyse the level of coverage.

\section{B. Scenario description}

In order to find a relation between quality of grass coverage and RGB values obtained by a TCS3472, different grass plots are used. Our tests are performed in "El Encin" facilities which belongs to the "Instituto Madrileño de Investigación $y$ Desarrollo Rural, Agrario y Alimentario (IMIDRA)” in Alcalá de Henares (Madrid, Spain. In these facilities, researchers are performing several studies focused on the water requirements of different grass species. They use plots of $1 \times 1.5 \mathrm{~m}$. which contains a combination of different grass species. A total of 15 plots have been selected according to the established conditions. The plots are divided into three different categories: HC, LC and VLC. From these 15 plots, 6 are considered as $\mathrm{HC}, 6$ as LC and 3 as VLC.

These images are analysed and the RGB values are obtained. The result is 3 matrixes of $100 \times 150$ values between 0 and 255 for each plot (one matrix for each colour). From these matrixes, we obtain the histogram to represent the gathered information and analyse it.

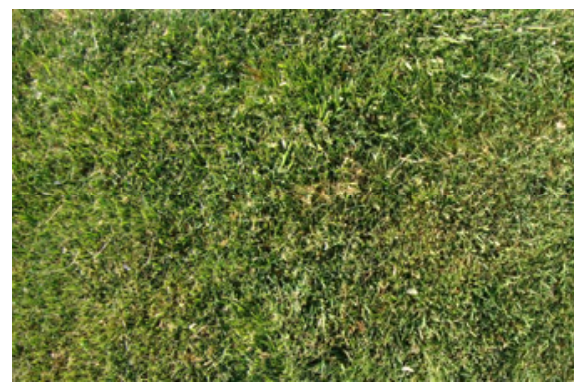

a)

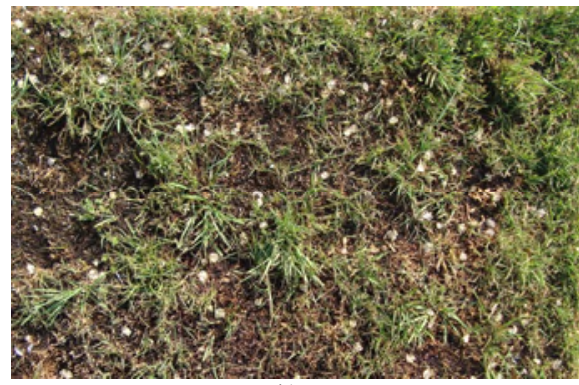

b)

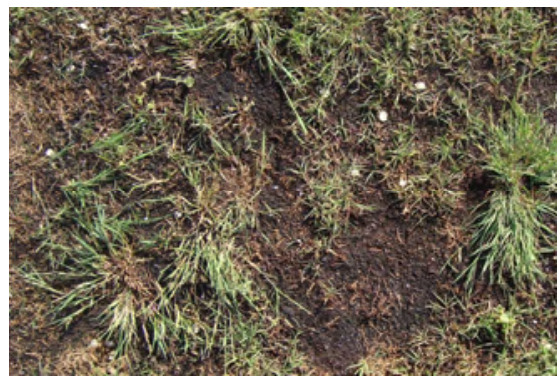

c)

Fig. 1. Examples of grass plots used in out experiment

\section{PROPOSAL}

This section presents the proposed system to gather the information about the grass described. First, the sensor and the node are described, the SAV and its components are shown. Finally, the operation process of our system is detailed.

\section{A. Proposed Architecture}

In order to develop an autonomous system capable of automating the irrigation lawns, we need to have a series of sensors to collect data and actuators to perform the necessary actions to maintain the correct growth of lawns (See Fig. 2).

Lawns count with several underground sprinklers that will rise. When irrigation is required, sprinklers will emerge from the ground due to the pressure of the water flowing through the underground water pipes beneath the lawn. In order to be sure that the SAV can freely move along the field to monitor the entire area, the sprinklers should be hidden underground. The SAV is located at its initial base. From this point, the SAV will start its route and will store the data in its local memory. At the end of the tour, the SAV will wirelessly send all the data to the coordinator node which will calculate the irrigation needs from the received data and previous experiences. All information is stored in a cloud on the Internet and allows remote access from any type of portable device [26].

\section{B. Adquisition system}

The data acquisition system is based on a small motorized electric vehicle that circulates over the lawn area to be measured or monitored (See Fig. 2). As the vehicle advances, it simultaneously takes 100 RGB values, in a line. These values are transmitted by $\mathrm{I} 2 \mathrm{C}$ bus to the Arduino Mega 2560 module.
The Arduino Mega 2560 module consists of 54 digital inputs/outputs, 16 analog inputs and $256 \mathrm{~KB}$ of memory for storing program codes.

To monitor the lawn and extract the RGB values, we have selected the TCS3472 color sensor [27] by AMS. This color sensor is composed by a matrix of $4 \times 3$ photodiodes where nine of them are equipped with color filters able to detect the light that affects them separating it according to an RGB color model. The remaining three LEDs do not contain any filter. The dynamic range of this sensor is $3.8 \mathrm{M}$ : 1 with a resolution of 16 bits per channel, a fast $\mathrm{I} 2 \mathrm{C}$ bus and it is able to generate an interruption signal or protection against electrical interference and flicker of illumination. The TCS3472 has no SYNC signal input.

The I2C protocol [28] is based on a master-slave architecture where a microcontroller, in our case the Arduino 2560 MEGA module, acts as master and can control a large number of slave devices. In our case the role of slave devices is assumed by the sensors. Our system uses 7-bit addresses. So, the microcontroller can address up to 128 devices.

The set of all these sensors are installed in the lower part of our SAV.The external aspect of the SAV is shown in Fig. 3. The SAV is connected to our network through a wireless connection based on the IEEE 802.11 protocol. The antenna is placed on the top of the SAV. This antenna will provide the capability of sending the collected data to the coordinator node. Moreover, in the top of SA, there is a solar panel that supplies energy to a battery that powers the system. The bottom of SAV also contains two deflectors which help us to remove any possible object that can interfere the measurement process. It is 
necessary to ensure that the data from the RGB sensors belongs only to the grass and not from other objects that can generate wrong results.

\section{Operation algorithm}

The vehicle begins its movement and advances to the first position. At this point, it takes the RGB values of its position. In each measure, the system makes an approximation to determine if there is any problem with the grass at that point in the area. If the values recorded are not the typical ones of a highly grass-covered area, the system will tag the point in the field where the problem was detected.

To know the position of the vehicle, it uses a system formed by an encoder that is responsible for counting the advance of the vehicle. The vehicle will check if it has reached the final position of the route. If the route has not been completed, the vehicle will move to the next position and perform a new measurement.

When the SAV reaches its final position, it sends the data to the coordinator node and checks similar results in the database. If these data are new, the system will estimate the amount of water and/or the amount of fertilizer required for the monitored area and this data will be stored in the database. If these values (or very similar ones) have been previously recorded, the system will take the calculations from the database. Finally the sprinklers will be activated to perform the irrigations. Fig. 4 shows the operating algorithm used by our irrigation system.

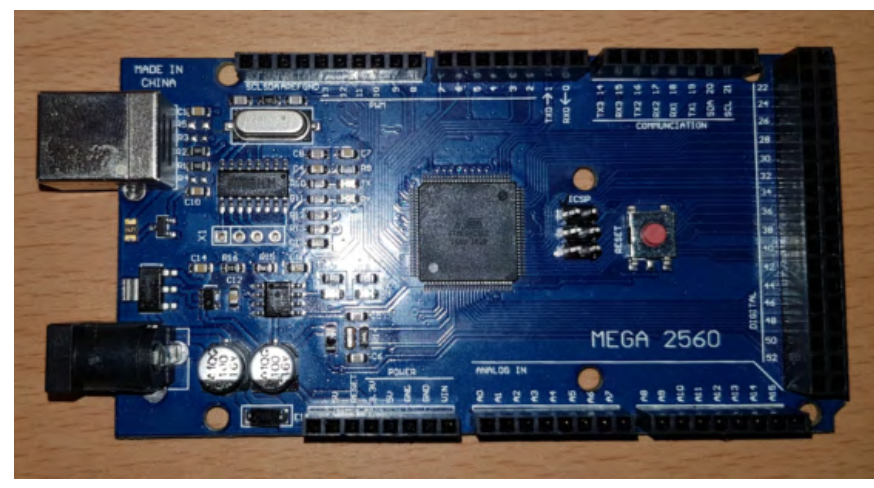

Fig. 2. Arduino Mega 2560 module used in our test

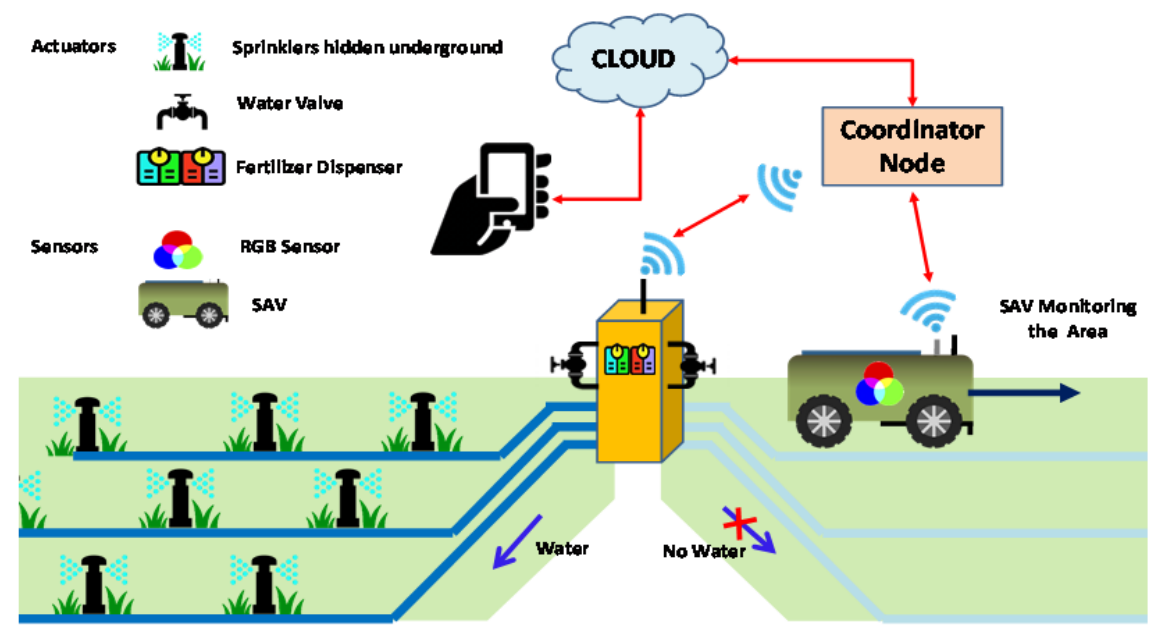

Fig. 3. Proposed architecture

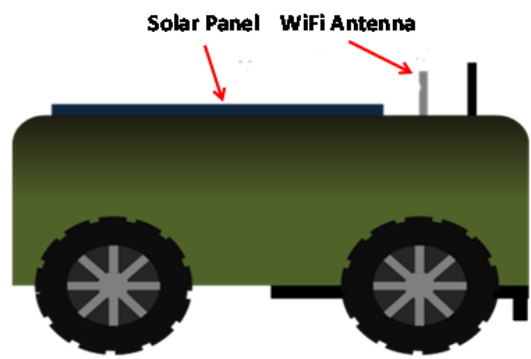

a) Lateral View

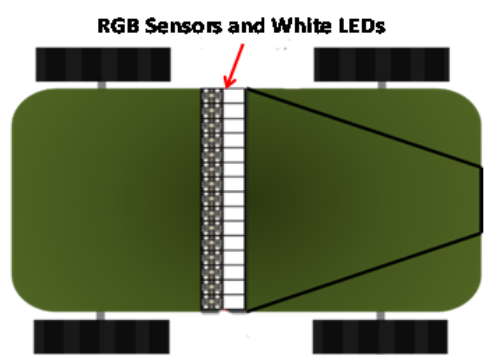

b) Bottom View

Fig. 4. SAV and its components 


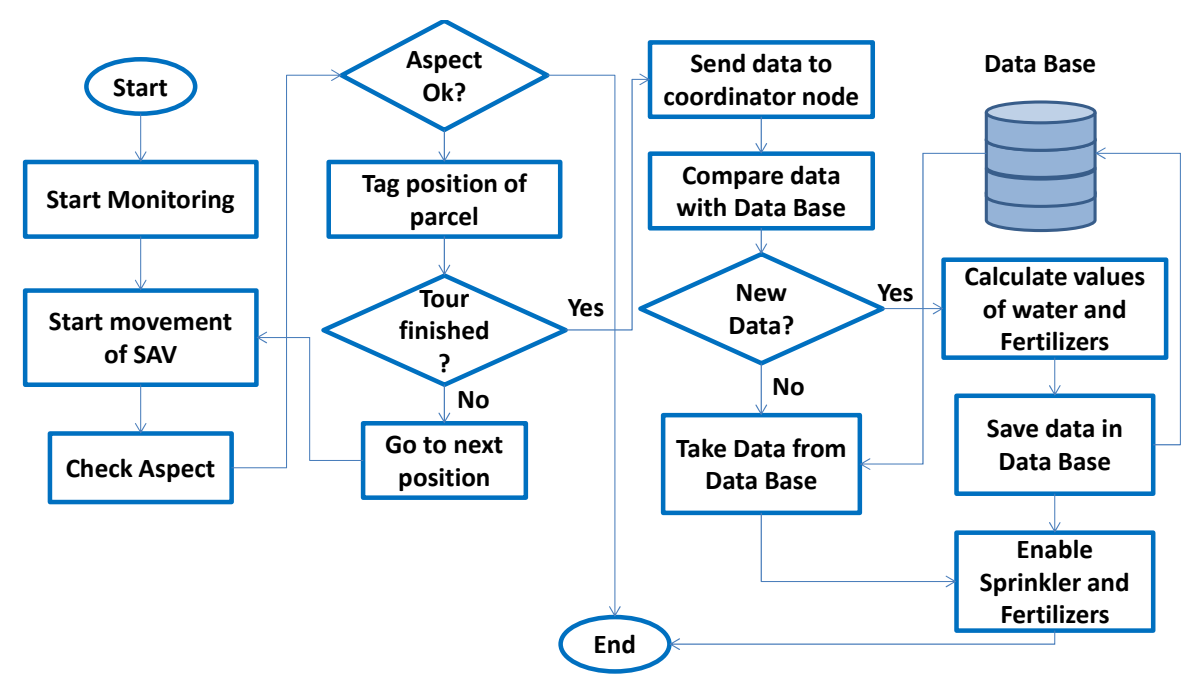

Fig. 5. Algorithm for data processing

\section{RESUlTS}

This section shows the statistical relation between the grass coverage and the RGB values. Moreover, the hypothesis will be confirmed or dismissed.

\section{A. Lawns clasification}

The first step is to obtain histograms of the $\mathrm{HC}, \mathrm{LC}$ and VLC plots. From each plot of $1.5 \times 1 \mathrm{~m}$, we have obtained a matrix of data with $150 \times 100$ values for each colour (red, green and blue). With this data, we obtain the histograms shown in Fig. 6, Fig. 7 and Fig. 8 which represent the brightness from 0 to 155 in the horizontal axis and the amount of pixel with this brightness in the vertical axis.

Fig. 6. shows the histogram obtained for the data from the Red colour. Green lines represent the histograms of the $6 \mathrm{HC}$ plots (from $\mathrm{HC} 1$ to HC6). The histograms of the 6 plots with LC, i.e., from LC1 to LC6, are represented in orange. Finally, the red lines refer the histograms of the VLC plots (from VLC1 to VLC3). With a naked eye, there is no relation between the different groups (HC, LC, and VLC) and the histograms. This absence of relation, diminish our first hypothesis. Thus, it is not possible to affirm that the areas with LC and VLC present higher values in red histogram.
Fig. 7 shows the histograms from the data that correspond to the Green colour. In this case, it is easy to observe that there is some relation between the histogram and the plots.

For the region of brightness from 40 to 60 , it is possible to distinguish between HC, LC and VLC. This relation should be studied. Other important aspect to highlight is that the values of VLC are lower than the values of $\mathrm{HC}$ and the peaks of the histograms from the VLC plots are placed between 64 and 92 of Brightness. In addition, the peaks of histograms from the LC plots are between 97 and 132. In the same interval the plots with $\mathrm{HC}$ present the peak of the histogram; however, the values of these peaks are higher in the plots with HC than in the plots with LC. This, corroborates our second hypothesis, the plots with higher coverage presents higher values in the green histogram.

Fig. 7 shows the data from the histograms obtained for Blue. Again, it is easy to identify a pattern. In fact, the histograms from the HC plots have lower values of brightness. The peaks of the histograms obtained for the $\mathrm{HC}$ plots between 45 and 65 of Brightness. However, in this case there is no difference between LC and VLC plots. Both, LC and VLC, have the peaks between 56 and 83 of Brightness. However, they present lower values than the $\mathrm{HC}$ plots. Our third hypothesis has been partially confirmed. It is only possible to distinguish $\mathrm{HC}$ from the rest of cases. However, it is not possible to distinguish between LC and VLC. 


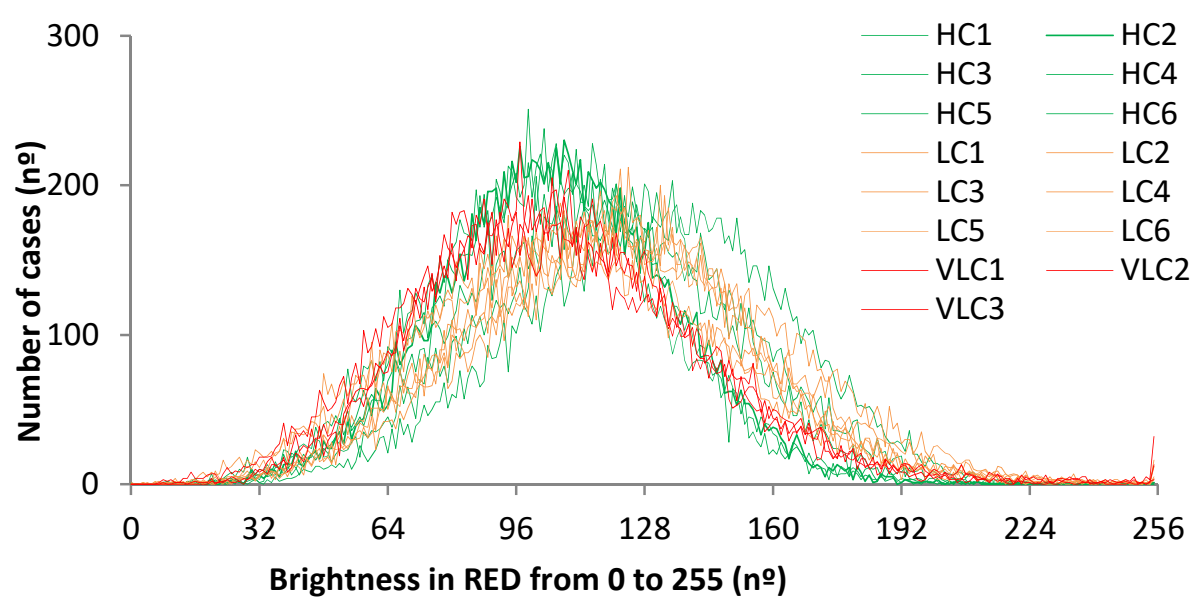

Fig. 6. Histograms Red

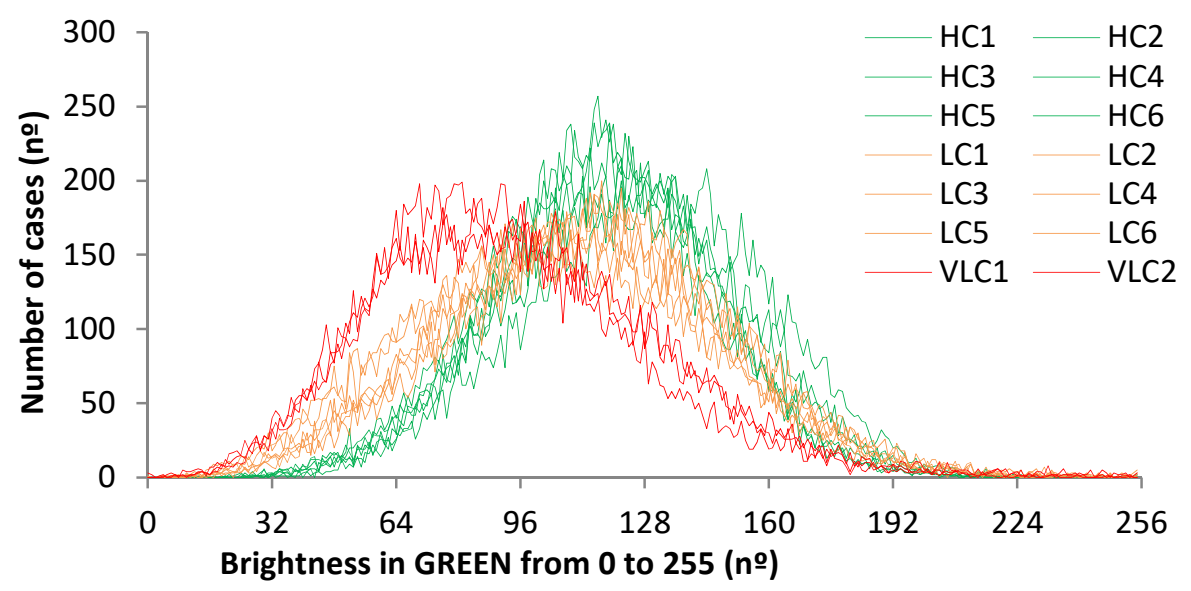

Fig. 7. Histograms for Green

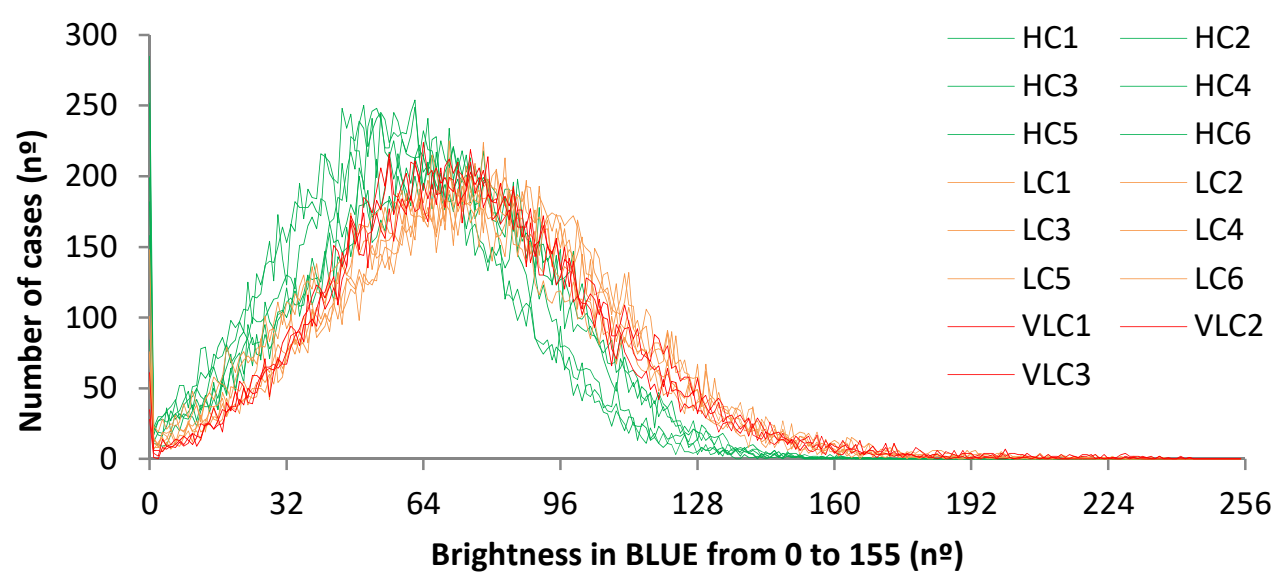

Fig. 8. Histograms for Blue

The next step is to perform a deeper study of the data from the green component of the RGB data.

Because the region of the histogram that appears the differences between HC, LC and VLC are from 40 to 60, we focus this study in this region. Fig. 9 shows a dendrogram with the results. The dendrogram is calculated with the method of the nearest neighbour and the squared Euclidean is used as a metric. To obtain this dendrogram, we have used the software
Statgraphics Centurion. This dendrogram demonstrates that the differences between groups observed in region 40 to 60 of the histogram are statistically significant. The dendrogram make the clusters according to the typology of the plots. This relation will be used in our SAV to distinguish between grass areas with $\mathrm{HC}, \mathrm{LC}$ and VLC.

Finally, we show the mathematic model we will use in our system. We propose to use the summation of the histogram 
values in the region from 40 to 60 , to determinate in which group each plot can be classified. From the matrix of 15.000 values, we have to extract the number of elements that have a value between 40 and 60 . This is matrix $A$ where $a_{i, i}$ represents each value of the green component of the RGB value (See Eq. (1)). To extract the data of interest, elements with values between 40 and 60 , the following operation must be applied to A. B is a matrix with the same number of columns and rows than A. It is shown in Eq. (2). Then, a subtraction operation between $\mathrm{A}$ and $\mathrm{B}$ is done as Eq (3) show. The result of this operation is known as $\mathrm{C}$. with the values of $\mathrm{C}$, we generate a new matrix called D using Eq. (4).

Now, we have a matrix with values of 0 or 1 where 0 represents the values lower than 40 and 1 represents the values greater or equal than 40 in the original matrix. The matrix E which contains the result of operations in matrix D is created (See Eq. (5)). The next step is a subtraction of matrix $\mathrm{E}$ to matrix A; the result of this operation is $\mathrm{F}$ and it is shown in Eq. (6).

Now, we generate a new matrix, $G$, based on the values of $\mathrm{F}$ as shown in Eq. (7). Now, we have a matrix with values of 0 or 1 , where 0 represents the values higher than 60 and 1 represents the values lower or equal than 60 in the original matrix.

The last step is to multiply the matrixes D and G. Its product gives as result the matrix $\mathrm{H}$. It is a matrix composed by values of 0 and 1 , where 1 represents the values between 40 and 60 and 0 represents the rest of values. The summation of $\mathrm{H}$ matrix of the analyzed plots is shown in Fig. 10.

$$
\begin{aligned}
& A=\left[\begin{array}{cccc}
a 1,1 & a 1,2 & \cdots & a 1,150 \\
a 2,1 & a 2,2 & \ddots & a 2,150 \\
\vdots & \vdots & & \vdots \\
a 100,1 & a 100,2 & \cdots & a 100,150
\end{array}\right] \\
& B=\left[\begin{array}{cccc}
39.5 & 39.5 & \cdots & 39.5 \\
39.5 & 39.5 & \ddots & 39.5 \\
\vdots & \vdots & \cdots & \vdots \\
39.5 & 39.5 & \cdots & 39.5
\end{array}\right] \\
& C=A-B \\
& D=\left[\begin{array}{ccc}
\frac{c 1,1}{|c 1,1|}+1 & & \frac{c 1,150}{2} \\
\vdots & \ldots & \frac{|c 1,150|}{2}+1 \\
\vdots \\
\frac{c 1,100}{|c 1,100|}+1 \\
\frac{\mid c}{2} & \ldots & \frac{c 100,150}{|c 100,150|}+1 \\
2
\end{array}\right] \\
& E=\left[\begin{array}{cccc}
60.5 & 60.5 & \cdots & 60.5 \\
60.5 & 60.5 & \ddots & 60.5 \\
\vdots & \vdots & \cdots & \vdots \\
60.5 & 60.5 & \cdots & 60.5
\end{array}\right] \\
& F=A-E \\
& G=\left[\begin{array}{ccc}
\frac{f 1,1}{-|f 1,1|}+1 & & \frac{f 1,150}{2} \\
\vdots & \ldots & \frac{-|f 1,150|}{2}+1 \\
\frac{f 1,100}{-|f 1,100|}+1 & & \frac{f 100,150}{2} \\
\frac{-|f 100,150|}{2}+1
\end{array}\right] \\
& H=D \times G
\end{aligned}
$$

It is possible to see that the results of plots with the same coverage are similar. The summation of plots with $\mathrm{HC}$ is lower than summation of plots with LC and VLC. Summations lower than 500 belong to plots with HC. Summation higher than 1500 belong to plots with VLC. With these thresholds, it is possible to classify the plot coverage using the RGB data.
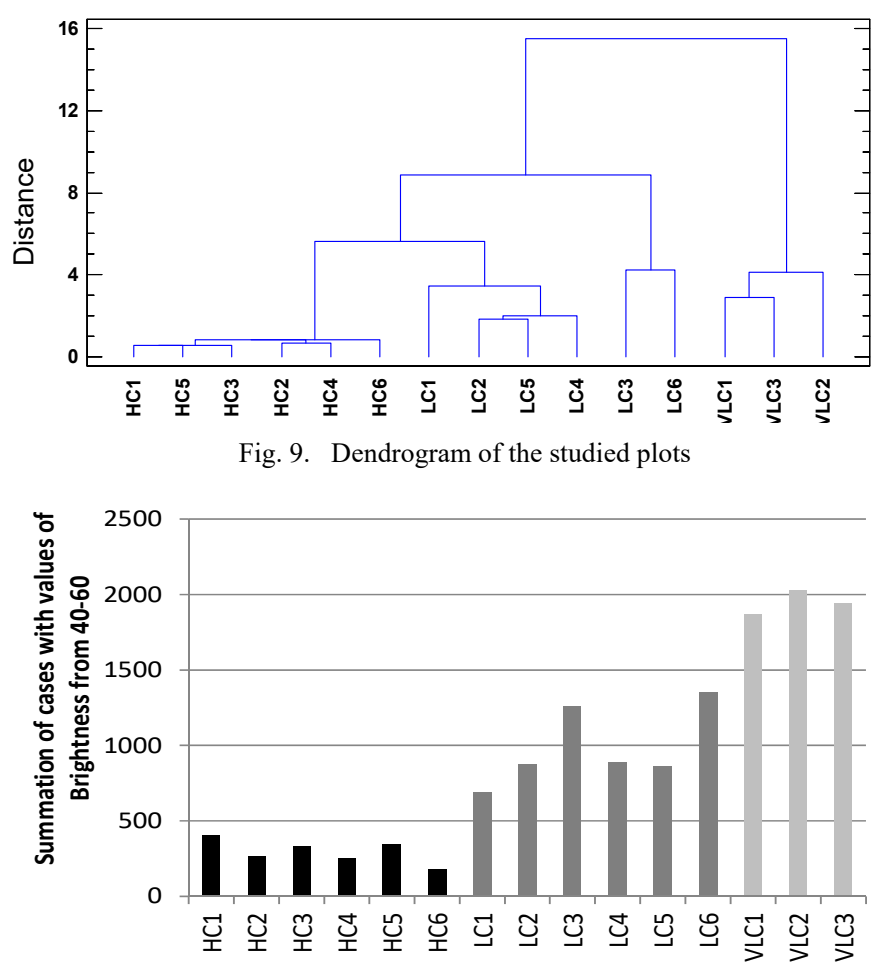

Fig. 10. Summation of cases with values of Brightness form 40 - 60

\section{CONCLUSIONS}

The concept of smart cities and how to develop new technology for achieving better sustainability in the use of resources are currently the important topic that are being investigated.

In this paper, we have designed a SAV that is part of a WSN for urban lawns monitoring in a scenario of smart cities. We have proposed an architecture composed by several sensors and actuators that interact with a coordinator node. This node is in charge of enabling a set of sprinklers to water the grass. The decisions made by this node are related to the sensors and the aspect of lawns, i.e., the level of coverage of each lawn.

To be able of monitoring the level of coverage, we have developed a SAV with a set of 100 RGB sensors. From the data sensors, we have processed the obtained values in order to separate the green component. Finally we have selected the range from 40 to 60 to classify the level of coverage of our lawns. After statistically analyzing the results we have observe that it is easy to detect the level of grass coverage and using this information to make estimations about the necessary water to maintain these lawns.

As future work, we would like to implement the whole network test the operation of the SAV with the rest of network 
to evaluate the autonomy of this system. Moreover we would like to study the possibility of extending this kind of systems to other areas.

\section{ACKNOWLEDGMENT}

This work has been partially supported by the "Ministerio de Educación, Cultura y Deporte", through the "Ayudas para contratos predoctorales de Formación del Profesorado Universitario FPU (Convocatoria 2014)". Grant number FPU14/02953.

\section{REFERENCES}

[1] The World's Water. Available online: https://water.usgs.gov/edu/earthwherewater.html (Last access: May 29, 2017)

[2] NASA. 2017. "The Importance of Freshwater." Https://pmm.nasa.gov/waterfalls/science/freshwater. (Last access: May 29, 2017) .

[3] C. J. Vörösmarty, P. Green, J. Salisbury, R. B. Lammers, "Global water resources: vulnerability from climate change and population growth," Science, vol. 289, no. 5477, pp. 284-288, 2000.

[4] G. C. Lazaroiu and M. Roscia, "Definition methodology for the smart cities model,” Energy, vol. 47, pp. 326-332, 2012.

[5] L. Parra, S. Sendra, J. Lloret, I. Bosch, "Development of a conductivity sensor for monitoring groundwater resources to optimize water management in smart city environments," Sensors, vol. 15, no. 9, pp.20990-21015, 2015.

[6] L. Gabrielli, M. Pizzichini, S. Spinsante, S. Squartini, R. Gavazzi, "Smart water grids for smart cities: A sustainable prototype demonstrator," In Proceedings of the 2014 European Conference on Networks and Communications (EuCNC), Bologna, Italy, 23-26 June 2014; pp. 1-5.

[7] V. S. Kumar, I. Gogul, M. D. Raj, S. K. Pragadesh, J. S. Sebastin, "Smart Autonomous Gardening Rover with Plant Recognition Using Neural Networks," Procedia Computer Science, vol. 93, pp. 975-981, 2016.

[8] A. K. Tripathy, A. Vichare, R. R. Pereira, V. D. Pereira, J. A. Rodrigues, "Open source hardware based automated gardening system using lowcost soil moisture sensor," In In Proceedings of the 2015 IEEE International Conference on Technologies for Sustainable Development (ICTSD), Mumbai, India, 4-6 February 2015, 2015; pp. 1-6.

[9] T. Okayama, "Future Gardening System-Smart Garden," Journal of Developments in Sustainable Agriculture, vol. 9, no. 1, pp. 47-50, 2014.

[10] R. Aziz, Consumption of Energy and Routing Protocols in Wireless Sensor Network, Network Protocols and Algorithms, 2016, Vol 8, No 3. Pp. 76-87.

[11] S. Yadav, K. Swarnkar, T. Chauhan, R. Jain, "How to improve food security and farming systems by using wireless sensor networks (WSN)," Journal of Information Engineering and Applications, vol. 2, no.6, pp. 11-20, 2012.

[12] L. Ruiz-Garcia, L. Lunadei, P. Barreiro, I. Robla, “ A review of wireless sensor technologies and applications in agriculture and food industry: state of the art and current trends," Sensors, vol. 9, no. 6, pp. 4728-4750, 2009.

[13] J. Marín, J. Rocher, L. Parra, A. Plaza, P. V.Mauri, J. Ruiz-Fernández, S. Sendra, J. Lloret, “Automatización en la caracterización del cultivo de céspedes en praderas urbanas" In In Proceedings of the IX Congreso Ibérico de Agroingeniería, Bragaça, Portugal, 4 - 6 September, 2017.

[14] H. Khaleeq, A. Abou-elnour, and M. Tarique, "A Reliable Wireless System for Water Quality Monitoring and Level Control," Network Protocols and Algorithms vol. 8, no. 3, pp. 1-14, 2016.

[15] V. S. Kumar, I. Gogul, M. D. Raj, S. K. Pragadesh, and J. S. Sebastin, "Smart Autonomous Gardening Rover with Plant Recognition Using Neural Networks," Procedia Computer Science, vol. 93, no. September, pp. 975-981, 2016.

[16] A. K. Tripathy, A. Vichare, R. R. Pereira, V. D. Pereira, and J. A. Rodrigues, "Open source hardware based automated gardening system using low-cost soil moisture sensor," In Proceedings of the 2015 International Conference on Technologies for Sustainable Development (ICTSD) 4th to 6th February, 2015, Mumbai, India, pp. 1-6.

[17] A. Macedo-Cruz, G. Pajares, M. Santos, and I. Villegas-Romero, "Digital image sensor-based assessment of the status of oat (Avena sativa L.) crops after frost damage," Sensors, vol. 11, no. 6, pp. 60156036, 2011.

[18] C. Yang, "A high-resolution airborne four-camera imaging system for agricultural remote sensing," Computers and electronics in agriculture, vol. 88, pp. 13-24, 2012.

[19] J. Lloret, I. Bosch, S. Sendra, and A. Serrano, "A wireless sensor network for vineyard monitoring that uses image processing," Sensors, vol. 11, no. 6, pp. 6165-6196, 2011.

[20] A. J. Garcia-Sanchez, F. Garcia-Sanchez, and J. Garcia-Haro, "Wireless sensor network deployment for integrating video-surveillance and datamonitoring in precision agriculture over distributed crops," Computers and Electronics in Agriculture, vol. 75, no. 2, pp. 288-303, 2011.

[21] S. Candiago, F. Remondino, M. De Giglio, M. Dubbini, and M. Gattelli, "Evaluating multispectral images and vegetation indices for precision farming applications from UAV images," Remote Sensing, vol. 7, no. 4, pp. 4026-4047, 2015.

[22] C. Cambra, J. R. Díaz, and J. Lloret, "Deployment and Performance Study of an Ad Hoc Network Protocol for Intelligent Video Sensing in Precision Agriculture," In proceedings of the International Conference on Ad-Hoc Networks and Wireless (ADHOC-NOW 2014), 22-27 June 2014, Benidorm, Spain, pp. 165-175.

[23] L. García Torres, J. M. Peña-Barragán, F. López-Granados, M. JuradoExpósito, and R. Fernández-Escobar, "Automatic assessment of agroenvironmental indicators from remotely sensed images of tree orchards and its evaluation using olive plantations," Computers and Electronics in Agriculture, vol. 61, no. 2, pp. 179-191, 2008.

[24] BOE, "Real Decreto-ley 8/2014, de 4 de julio, de aprobación de medidas urgentes para el crecimiento, la competitividad y la eficiencia.," Boletín oficial del estado, pp. 52544-52715, 2014.

[25] D. J. Mulla, "Twenty five years of remote sensing in precision agriculture: Key advances and remaining knowledge gaps," Biosystems engineering, vol. 114, no. 4, pp. 358-371, 2013.

[26] C. Cambra, S. Sendra, J. Lloret, L. Garcia, " An IoT Service-Oriented System for Agriculture Monitoring". In proceedings of the 2017 IEEE International Conference on Communications (ICC 2017), May 21-25, 2017, Paris (France).

[27] TCS3472 features. Available at: https://ams.com/jpn/content/download/319364/1117183/file/TCS3472 _Datasheet_EN_v2.pdf. (Last access: May 29, 2017)

[28] I2C protocol features. In Texas Instrument website. Available at: http://www.ti.com/lit/an/slva704/slva704.pdf. (Last access: May 29, 2017). 